\title{
Flaws in the Medical Literature: Who's to Blame?
}

\author{
Jerome P. Kassirer
}

Received: 4 August 2010/Accepted: 27 August 2010/Published online: 26 September 2010

(C) L. Hirszfeld Institute of Immunology and Experimental Therapy, Wroclaw, Poland 2010

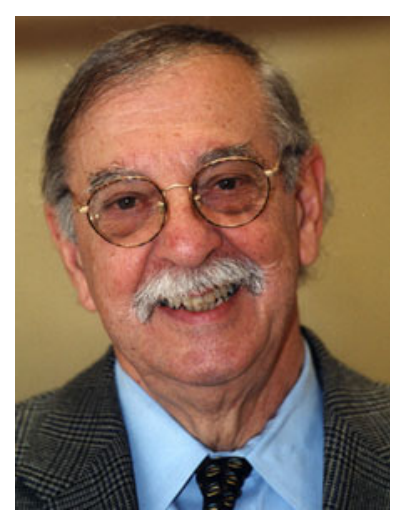

Jerome P. Kassirer, MD, is Distinguished Professor and Senior Assistant to the Dean at Tufts University School of Medicine and Visiting Professor at Stanford University. In editorials in the New England Journal of Medicine, and in multiple publications elsewhere, he has promoted professionalism, ethical scientific conduct, patient involvement in decision making, appropriate use of firearms, reliable assessment of the quality of healthcare, and rational approaches to medical diagnosis and decision-making. He was been critical of for-profit medicine, the abuses of managed care, political intrusions into medical decisions, and financial conflicts of interest. His 2004 book, on financial conflict of interest in medicine, "On The Take: How Medicine's Complicity With Big Business Endangers Your Health," was published by Oxford University Press and his 2009 book, "Learning Clinical Reasoning" was published by Lippincott.

J. P. Kassirer $(\varangle)$

School of Medicine, Tufts University,

136 Harrison Ave., Boston, MA 02111, USA

e-mail: jpkassirer@aol.com
Dr. Kassirer served as Editor-in-Chief of the New England Journal of Medicine (1991-1999), on the American College of Physicians' Board of Governors and Board of Regents, chaired the National Library of Medicine's Board of Scientific Counselors, and is a past Chairman of the American Board of Internal Medicine. He is a member of the Board of Directors of the National Committee for Quality Assurance and Open Medicine. He has been elected to the Association of American Physicians, the Institute of Medicine of the National Academy of Sciences, and the American Academy of Arts and Sciences.

The litany of complaints is now well known, and seems endless. Pharmaceutical companies have hired medical writers to write papers favorable to their marketing aims and have paid academic physicians or key community opinion leaders to sign on as authors. The same companies have engaged physicians to join their speaker's bureaus, supplied them a nice honorarium and pretty slides that show a product in good light, and have continued to use them as speakers as long as they promote the company's message. Companies have paid physicians to come to "educational" half day seminars that are scarcely disguised marketing efforts (the rest of the day the doctors play golf). Some company executives have intimidated physicians who are honestly speaking out about the complications of one of the company's products. Companies have designed studies that are almost certain to get positive results. Physicians have published studies and failed to disclose that they were company-paid speakers or consultants, or paid for enrolling patients. Industry has hidden negative studies. Company executives have intentionally allowed their marketing departments to promote drugs that they knew were ineffective, had dangerous side effects, or both. In making arrangements with academic investigators to run 
their clinical trials, companies have declared that the study results belong to them and they have insisted on keeping all the reported data, carrying out all the statistical manipulations, and providing the principal investigator with completed figures and tables. Moreover, the companies have often reserved the right to approve the final manuscript.

The titles of books and articles echo these damning accusations. "Industry-sponsored research: A broken system." "The truth about drug companies: How they deceive us." "Bias, spin, and misreporting: Time for full access to trial protocols and results." "Rent a researcher." "Selling sickness: The pharmaceutical industry and disease mongering." "Ghost management: How much of the medical literature is shaped behind the scenes by the pharmaceutical industry?" "Authors, ghosts, damned lies, and statisticians." "Ghostwriting: the dirty little secret of medical publishing that just got bigger." "The trouble with medical journals." "Medical journals are an extension of the marketing arm of pharmaceutical companies." "Prevalence of articles with honorary authors and ghost authors in peerreviewed medical journals."

There is little doubt that drug, device, and biotechnology company policies, executives, and marketing managers share a great deal of the blame for flaws in our medical information. Until recently we had little reason to distrust the data from these companies. They produced many new and effective tests and therapies, and many were among the most admired and trusted companies in the world. Yet, in information uncovered in the course of litigation, mostly in England and the United States, many industry practices emerged since the late 1990s that favored the widespread marketing of drugs, overstated the benefits and understated the risks of products, and put the public at risk. Profits often seemed to trump truth.

The medical profession was quick to look outside of itself for an explanation; it was the big, bad companies' fault that the medical information that physicians use every day had been corrupted. If a loss of trust exists, the argument went, blame it on industry. Yes, industry was to blame, but so was the profession; many had been corrupted by the financial arrangements they accepted from an industry intent on making the profession a marketing outreach of its staff.

A 3-year investigation led me to believe that the profession was substantially culpable. In virtually every endeavor that involved physicians, I found evidence that physicians shared the responsibility for faulty data. Industry-written papers could not have been published under the name of a prominent physician if the doctor had refused to become a ghost author. Clinical trials of drugs are necessarily sponsored by industry, but they cannot be run without academic investigators and hundreds of physicians in the community, and physicians on institutional review boards must approve their protocols. Study designs could not be manipulated to yield positive results unless the physicians involved had agreed not only to participate, but to cede authority on the final manuscript to the company. Many company strategies for hiding data would not have worked if physicians on drug company boards had been more inquisitive or more objective. Many exaggerations of benefits and undermining of risks might have been avoided if medical editors had scrutinized industry-sponsored trials more carefully. And false claims about drugs might never have been uttered in continuing medical education sessions if physicians demurred when invited to become members of company speaker's bureaus, or if physicians refused to attend continuing medical education courses when everyone knew that they were biased.

Cleary there was extensive complicity between physicians and industry, and it has not abated entirely. What were the physicians who allowed and even perpetrated bias thinking? Were the rewards of a continuous supply of drug company clinical trial money so large? Was the personal income for speaking and ghostwriting so huge? For some, undoubtedly money was a factor, but many were either not paying sufficient attention to what they were saying or signing, and many probably thought that a slight bias did not matter. Even if an introduced bias has no influence on patient care, any bias is worth trying to avoid. Objectivity and reliability must be the hallmark of any science, including the scientific basis of medical practice.

I believe that for the most part, biases introduced by most physicians were inadvertent and not conscious actions. I believe that few violate their oath to be mindful of their obligation to patients, but the payments probably had a subconscious influence that allowed them not to live up to professional maxims.

In the United States we have come a long way in dealing with some of the problems described here. Clinical trials must be registered, a policy that allows investigators to assess whether a trial has been published. Editors insist that authors follow the authorship standards of the International Committee of Medical Journal Editors or the World Association of Medical Editors, both of which specify that an author must be able to take responsibility for the content of the manuscript and define an author as one who has made substantive contributions to the conception and design of a study and participated actively in the preparation of the manuscript. Many medical centers, universities, and physician organizations have introduced new financial conflict of interest rules, and by the year 2013 the US federal government will require all pharmaceutical companies to report anything more than a trivial amount of money or gifts that they give to any physician. These restrictions have been helpful, but they are not likely to 
completely erase errors, intentional lapses of integrity, or complicity by executives to market faulty drugs and devices. And therefore they are not likely to restore the reputation of either the drug and device industry or the profession.

A principal approach to deal with the financial conflicts of interest within the profession is disclosure of the funds provided by companies to individuals. In fact, the US law that will be implemented in 2013 offers disclosure as its exclusive tool. Unfortunately, disclosure is only a partial solution. Even though the principal criticism of physicians over the past decade has been their failure to disclose financial relations with industry, disclosure is a only necessary step, not a sufficient solution. It is not the failure of disclosure that is the problem: it is the financial conflict itself that promotes bias. For this reason, the only way to protect against bias is to eliminate or neutralize the conflict, not just to disclose it.

Public trust is difficult for a profession or a company to achieve, but trust is easy to lose. Only a small incident in which the public perceives that a profession or industry is acting in its own best interests can destroy decades of good will. The government can play a role in restoring trust, but in the final analysis it is the profession that must show that its fundamental tenets favor patients, not itself. Conflict of interest requirements must go beyond disclosure to avoid the reality and the perception that some physicians care more about their personal income than they do about optimal patient care.

Restoring trust in the evidence base of medicine is a moral imperative. If the profession is unable to do so, government is likely to impose many more regulations on research efforts and publishing. Industry must be forced to put the health of the public ahead of quarterly profits. Fines for failing to do so have had little effect in preventing companies from violating their own standards. Perhaps it is time to hold individual industry executives responsible when they knowingly put the public at risk. 